\title{
Habits and Goals in Human Behavior: Separate but Interacting Systems
}

\author{
Wendy Wood, ${ }^{1}$ Asaf Mazar, ${ }^{2}$ and David T. Neal ${ }^{3}$ \\ 1 Department of Psychology and Marshall School of Business, University of Southern California \\ 2 \\ Department of Psychology, University of Southern California \\ 3 Catalyst Behavioral Sciences and Duke University
}

\begin{abstract}
Author Note
We have no conflicts of interest to disclose. The authors are grateful to Claire Gillian, Merel Kindt, Trevor Robbins, Sanne de Wit, and Poppy Watson for their insightful comments on an earlier version of this article and Jan De Houwer for graciously providing data from his research. Correspondence concerning this article should be sent to Wendy Wood, Department of Psychology, University of Southern California, Los Angeles, CA 90089 email: wendy.wood@usc.edu
\end{abstract}




\begin{abstract}
People automatically repeat behaviors that were frequently rewarded in the past in a given context. Such repetition is commonly attributed to habit, or associations in memory between a context and a response. Once habits form, contexts directly activate the response in mind. An opposing view is that habitual behaviors depend on goals. However, we show that this view is challenged by the goal independence of habits across the fields of social and health psychology, behavioral neuroscience, animal learning, and computational modeling. It also is challenged by direct tests revealing that habits do not depend on implicit goals. Furthermore, we show that two features of habit memory-rapid activation of specific responses and resistance to change - explain the different conditions under which people act on habit or pursue goals Finally, we test these features with a novel secondary analysis of action-slip data. We find that habitual responses are activated regardless of goals, but can be performed in concert with goal pursuit.
\end{abstract}

Keywords: habit, action slips, goal pursuit, behavior change 


\section{Habits and Goals in Human Behavior: Separate but Interacting Systems}

An esteemed professor, perhaps thinking about an important research project, tries to enter his office building through the doorway he has used for years, despite knowing that the entry was recently closed for renovations. On realizing his mistake, he alters course toward a still-open doorway (example from Kruglanski \& Szumowska, 2020). This scenario reflects a central question in action control: How do habits and goals guide action selection and performance?

The answer you give to this question depends on your definition of habit. In most modern accounts of habit, habitual responses are directly cued by contexts (de Wit, 2017; Gardner, 2015; Knowlton \& Diedrichsen, 2018; Wood \& Rünger, 2016). The familiar cues as the professor walked through campus triggered taking the habitual, but now blocked, path. Upon encountering the barrier, conscious goal-pursuit was activated, and he overrode his habitual response and chose a new way into the building.

Yet some researchers have recently proposed that "all human actions are driven by specific goals," with the result that "habitual behavior is goal-driven," and designed to achieve valued behavioral outcomes (Kruglanski \& Szumowska, 2020, p. 4, see also De Houwer et al., 2018; Ainslee, in press). In this view, the professor's overlearned behavior persisted because a residual goal to use the old entrance overrode his goal to use the new entrance.

As we show in this article, the view that all behavior (even habits) must be goal-driven is intuitively compelling because it fits with people's well-entrenched belief in their own agency. However, this alternative account suffers a major flaw: In practice, it is not falsifiable. Research might successfully demonstrate goal independence of overlearned behavior by accounting for one, two, or a hundred goals. However, a near-endless supply of other goals could be inferred post hoc. Thus, in practical terms, the notion that all behavior is goal-driven is not open to 
empirical test; ruling out dependence on one goal leaves other (as yet untested) goals that could be at play. As even one proponent acknowledged, this argument has the downside of creating an “irrefutable claim that the behavior must be mediated by some type of goal" (De Houwer, 2019, p. 4).

Like many scientific controversies, this debate is unlikely to be resolved through pejorative labels or straw-person caricatures of modern habit theory (e.g., "purposeless action," “an empty class," "void of sense," Kruglanski \& Szumowska, 2020, pp. 2-3). It also will not progress through a false dichotomy implying that behavior must be either completely goaldirected or completely habitual. In the history of science, such dichotomies (e.g., nature vs. nurture, situation vs. person) have rarely been resolved by critical tests showing that one process wins over another. Instead, scientific progress in this area would benefit from empirical tests examining how and when behavior is guided by one process or the other, as well as how habits and goals interact.

In this spirit, we begin by explaining the tensions between habit and goal accounts of action in the history of psychology. We then review the extensive, diverse evidence from multiple subfields (e.g., animal learning, cognitive science, human neuroscience, behavior prediction) showing that habits are directly triggered by context cues (e.g., external settings, time of day, prior actions in a sequence) in ways that do not require the activation of goal states. As we explain, habit automaticity differs from the goal-dependent automaticity often studied in social psychology. Nonetheless, in evidence that habitual responding involves a separate process from goal pursuit, we identify the different moderating conditions that promote each type of action control. Finally, we report a new analysis of existing data that supposedly showed the goal-dependence of habit. Instead, the findings show evidence for the separate but interacting effects of habits and goals on behavior. 


\section{Evolution of the Habit Construct}

William James (1914) made the prescient argument that habits differ from conscious will in that habitual "action goes on of itself" (James, 1914, p. 42). Specifically, he observed that "a sequence of mental action which has been frequently repeated tends to perpetuate itself, so that we find ourselves automatically prompted to think, feel, or $d o$ what we have before been accustomed to think, feel, or do, under like circumstances, without any consciously formed purpose or anticipation of results" (italics in original, p. 24). Nonetheless, he noted that goals can set a habit in motion or correct habitual responses when they go awry. Thus, James's view of habit aligns with the direct context cuing account that prevails today across most of psychology.

In the following decades, researchers took more partisan views, prioritizing either habits or conscious will. Early behaviorists embedded the habit concept in mechanistic models of stimulus-response learning that treated all action as a product of environmental control (Hull, 1943; Skinner, 1938). This approach was quickly challenged by Tolman's (1948) purposive behaviorism in which all action is motivated by flexible mental representations-a perspective that laid some groundwork for the ensuing cognitive revolution. Social psychologists in particular embraced the Gestalt roots of Tolman's (1948) theorizing and replaced the behaviorists' stimulus-response mechanisms with motivational models of how people coordinate actions within their environment (Campbell, 1963). Even today, some social psychologists subsume habits within goal-dependent action (De Houwer, 2019; Kruglanski \& Szumowska, 2020).

Contemporary research, however, has revealed characteristic features of habit formation and performance that distinguish it in principled ways from goal-dependent action (de Wit, 2017; Gardner, 2015; Knowlton \& Diedrichsen, 2018). In brief summary of the emerging consensus, 
habits are formed largely through instrumental learning. ${ }^{1}$ As people repeat a rewarded action in a stable context, they incrementally develop associations in procedural memory between the response and recurring cues in that context (Wood \& Rünger, 2016). ${ }^{2}$

Once formed, habits are directly brought to mind by context cues without the need for recruiting the goal that may have motivated initial learning (or any other goal). This direct cuing is possible because habits are guided by cached representations in memory that store direct cueresponse associations (Haith \& Krakauer, 2018). In other words, the habit system generates behavior by directly activating response units (de Wit, 2017). Once the response is triggered in mind, people tend to act on it through ideomotor processes whereby the thought of a behavior brings about the corresponding physical response in a reflexive, automatic manner (James, 1898/2007). However, habitual action is not obligatory—given sufficient motivation and opportunity, people can override the response in mind.

In modern theorizing, then, habits are not goal-dependent, in that they are activated directly by context cues without supporting goals (de Wit, 2017; Knowlton \& Diedrichsen, 2018; Wood \& Rünger, 2016). Thus, people can act on habits independently from their current goals. Nonetheless, habits can still be goal-aligned, in that they yield a future desired state without

\footnotetext{
${ }^{1}$ People also may form habit associations from repeated contiguity, or the simple co-occurrence, of contexts and responses (K. J. Miller et al., 2019).

${ }^{2}$ Procedural memory, which underlies knowledge of how to perform tasks, while not being accessible to conscious introspection, supports the expression of skills as well as repetition of habits (Gupta \& Cohen, 2002).
} 
depending on goals as underlying causal drivers. ${ }^{3}$ For example, a seatbelt-wearing habit could be aligned with a goal of safety, but this does not mean that people, consciously or unconsciously, activate a safety goal each time they habitually reach for a seatbelt.

Although these basic features of habit performance are broadly accepted, the field is by no means monolithic in its treatment of habit. To avoid overclaiming a unified perspective, we note that researchers have adapted this basic model in diverse ways. For example, habits may reflect the formation of hierarchical action sequences in which a prior action directly cues a subsequent one (Dezfouli \& Balleine, 2013), and habits may be additionally propelled by Pavlovian instrumental transfer (Wiltgen et al., 2013). Still another line of work equates habit with model-free choices, reflecting cached knowledge from experience (Dolan \& Dayan, 2013). ${ }^{4}$ These different programs have emphasized different features of habit performance and revealed that habit characteristics do not always cluster together (Foerde, 2018).

As another caveat, readers should note that, for simplicity, we often refer to habits as either strong or weak, despite that habit memories develop gradually with repetition, showing no

\footnotetext{
${ }^{3}$ Note that, for clarity, we refer to such behaviors as "goal aligned," instead of Moors and De Houwer's (2006) more ambiguous term for our purposes, "goal directed." We thank an insightful reviewer for this suggestion.

${ }^{4}$ Still other work differs from our central model by equating habit with automaticity in general, thereby overlooking its distinguishing features (e.g., Hommel, 2019). Also worth note is research that locates habits in episodic retrieval of stimulus-response episodes, thereby conflating recency and frequency of responding (Giesen et al., 2019) — despite that recency is held constant in most experiments that manipulate habit in terms of practice frequency.
} 
categorical demarcation between weak and strong habits (Lally et al., 2010). In addition, habits do not function in isolation but integrate with other psychological processes in guiding behavior, especially for complex behaviors with multiple steps (e.g., going to the gym). For example, habits can integrate with conscious goal pursuit, as when goal pursuit boosts performance of desirable habits and inhibits undesirable ones (O’Reilly et al., 2020; Wood \& Neal, 2007). Nonetheless, for ease of presentation, we refer to responses as more or less habitual.

This central feature of habit performance-direct context-response cuing without requiring a corresponding goal — is supported widely by research. The next section of the article summarizes this research from multiple fields and then explains how habit differs from the goaldependent automaticity often studied in social psychology.

\section{Habits Are Directly Activated by Recurring Context Cues}

\section{Behavior Prediction Studies}

The independent influence of habits and goals in guiding behavior is a cornerstone of behavior prediction research. As Triandis (1977) argued, people act on their intentions (behavioral goals) when their habits are weak; however, when people have formed strong habit associations in memory, these guide behavior with minimal intentional control. This hypothesis has since been supported with everyday behaviors ranging from recycling to eating, physical activity, and safe sex (see meta-analyses by Gardner, 2015; Ouellette \& Wood, 1998).

A key finding in behavior prediction studies is that, regardless of how they are measured, explicit goals are poor predictors of strongly habitual behavior. That is, when participants had formed strong habits to take the bus, they continued to do so regardless of how they described their bus-riding intentions (e.g., reducing my carbon footprint vs. getting to school) or the level of abstraction used (e.g., catching the 9:45 shuttle vs. using public transit, Ji \& Wood, 2007). Thus, the behavior prediction findings are not due to failures to appropriately assess people's 
behavioral goals. However, the relative influence of habits and goals is more nuanced than the simple interaction demonstrated in behavior prediction studies, and as we show, it depends on a variety of moderating factors (see section entitled, "Moderators of Habit Performance").

\section{Behavioral Neuroscience of Habit}

A central finding in behavioral neuroscience is that the brain circuits activated during habit performance are separate from, but interconnected with, those associated with goaldependent actions. Yin and Knowlton's (2006) landmark analysis of basal ganglia function identified distinct cortico-basal ganglia networks mediating actions and habits, respectively. Specifically, as habits form through instrumental learning, the cortical networks associated with action control shift from the goal-driven associative network to the habit-based sensorimotor cortico-basal ganglia network.

Empirical evidence that habit-related neural systems are activated by repeated task performance without necessarily implicating goal-related neural systems comes from a metaanalytic review of neuroimaging (fMRI) studies during repeated performance at various tasks (i.e., sequential decision making, motor sequence learning, outcome devaluation of instrumental tasks; Patterson \& Knowlton, 2018). After extended practice at these tasks, activation peaked in the lateral putamen, an area associated with habitual responding, but not the caudate, an area associated with declarative memory involved in conscious goal pursuit. These findings suggest a repetition-associated habit neural system that is engaged through repeated task performance.

Additional evidence of the neural basis of habit learning comes from patients with brain damage (Knowlton \& Diedrichsen, 2018). Damage to striatal regions (especially the putamen), as occurs with Parkinson's disease, impedes performance at serial reaction time tasks requiring habit learning, while the ability to learn these tasks in flexible, goal-driven ways, using declarative knowledge is retained (Foerde, 2018). Some addictive drugs, such as cocaine 
dependence, similarly disrupt functioning in the putamen and promote habitual responding

(Ersche et al., in press). In contrast, damage to the hippocampus or medial temporal lobes, as with amnesia, preserves habit learning but impedes flexible retention of declarative knowledge to guide goal pursuit. This pattern of double dissociation, whereby habit learning can be selectively impaired while goal pursuit is not and vice versa, cannot readily be explained by the goaldependent view of habit. Instead, these findings suggest that habit memory captures features of task learning separate from knowledge of desired outcomes.

\section{Animal Learning of Habits}

In animal learning research, the "general consensus is that parallel and competing circuits exist in the brain for habits and goal(s)" (Amaya \& Smith, 2018, p. 145). In support, habit circuitry at the cellular level has been identified and shown to interact with separate circuitry for goal-mediated behavior (O’Hare et al., 2018). Other lines of research have shown that repetition is responsible for shifting the neural control of flexible, goal-dependent behaviors to the less goal-sensitive habit system (Corbit, 2018).

In animals, similar to findings with brain damaged people, disruption of the dorsolateral striatum, a neural area corresponding to the human putamen, decreases habitual responding and promotes goal pursuit. That is, after disruption to habit systems, animals navigate by using goaldirecting spatial cues, respond flexibly to changes in action outcomes, and show increased variance in action structures (Amaya \& Smith, 2018).

Perhaps the canonical demonstration of the goal-independence of habit comes from animal learning research using the outcome devaluation paradigm. Specifically, rats extensively trained to press a lever for food continued to do so even when the food was no longer valued or expected (Dickinson, 1985). In daily life, this aspect of habit performance emerges in action slips 
of the kind that began our article (i.e., taking a familiar but blocked route; Reason, 2017). We provide new evidence of the contributing role of habits in a later section entitled, "Action Slips." Computational Models

Finally, the distinction between goal-mediated and habit-based responding is reflected in current computational models of behavioral control (e.g., Botvinick \& Plaut, 2004; K. J. Miller et al., 2019). Notably, the conceptual precursors to these computational models conceived of all actions as explicitly oriented toward attaining a goal (Carver \& Scheier, 1982; G. A. Miller et al., 1960). However, as these models evolved in response to the emerging behavioral and neural data discussed above, they generally shifted to postulate distinct learning algorithms and computational modules for goals and habits. For example, Sun et al.'s (2005) CLARION model used a rule-extraction algorithm to account for goal-based learning but a very different backpropagation algorithm to account for habit learning. Similarly, K. J. Miller et al. (2019) postulated a goal module that selects actions based on their outcome value and a separate habit module that selects actions that were frequently repeated in the past, regardless of outcome.

Computational accounts also identify the ways that goals and habits coordinate. One possibility invokes an arbiter module to weigh the strength of habits against the motivation of goals and other action drivers (K. J. Miller et al., 2019). Another allows for a continuum of interaction between these two systems (O’Reilly et al., 2020). Still another possibility envisions a speed/accuracy tradeoff between the fast but inflexible habit system and the slow-in-choiceselection but flexible goal system (Keramati et al., 2011). Thus, computational models of human action have generally coalesced around the view that habits and goals arise from distinct, albeit interacting, underlying mechanisms.

In summary, the multiple levels of analysis reflected in human behavior research, behavioral neuroscience, animal learning, and computational modeling all converge in showing 
that habitual responding and goal pursuit are characterized by separable patterns of learning, behavioral expression, and underlying brain systems. Specifically, habit memory is largely insensitive to current behavioral goals while nonetheless sensitive to context cues, it is associated with different neural substrates than goals, and it is effectively modelled computationally through a habit module that interacts with, but is separate from, goal-based action control.

\section{Automatic Goals in Social Psychology}

The extensive evidence that habit memory is insensitive to explicitly held goals has led to recent claims that habits must, instead, be guided by implicit goals, or needs outside of conscious awareness (Kruglanski \& Szumowska, 2020). However, research has already directly tested--and refuted--this possibility.

In this research, people persisted in acting habitually regardless of their implicit attitudes or goals. For example, participants with strong habits to watch TV or to recycle continued to perform those behaviors regardless of the automaticity of their attitudes toward TV watching or recycling, respectively (Ouellette \& Wood, 1998, accessibility assessed from reaction time to report attitudes). Similarly, hungry participants extensively trained in a food choice task to select carrots (thus forming habits to choose carrots), persisted in doing so even when they could have M\&Ms, and this effect was not due to implicit attitudes toward carrots or M\&Ms (Lin et al., 2016, implicit attitudes assessed with the Affect Misattribution Procedure). In addition, a standard procedure to manipulate nonconscious goals guiding speech volume did not influence participants with habits to speak loudly in a particular context (Neal et al., 2012).

Classic goal theory provides additional reason to differentiate habits from implicit goals. Automatic goals are thought to activate a variety of responses instead of being tied to one specific response (Kruglanski et al., 2002). Thus, a hungry person with an implicit goal for comfort might choose pizza, fried chicken, or pie. Even strongly desired goals that stably 
characterize people's motives are met flexibly through multiple substitutable behavioral means. To select from multiple means, people supposedly choose the one with the highest expected value to meet their current goals.

The different ways that implicit goals and habit memory guide behavior was illustrated in research that primed achievement goals (Hassin et al., 2009). After priming or not, all participants then repeatedly performed an instrumental task in which the correct choices changed several times without warning. As expected, participants implicitly primed with achievement goals flexibly learned the newly correct choices. Participants without goal priming, however, acted more on habit, persisting with responses that had been successful in the past, before the change. Thus, implicit achievement goals prompted flexible changes in behavior, whereas habitlike responses were rigid and insensitive to changes in outcome value.

In general, automatically activated goals appear to function similarly to explicit goals in promoting a strategic orientation to adapt to changing environmental demands. Demonstrating this similarity, manipulations of implicit and explicit goals to work hard were associated with comparable physiological changes (Takarada \& Nozaki, 2018). Implicit and explicit goals also have similar effects in guiding behavior (Bargh, in press). Suggesting additional connection, implicit goal pursuit depends, at least in part, on explicit reasoning; People act on implicitly activated goals and concepts that are attributed to their own thoughts (e.g., "I am thinking of money, so I must want to stick to my budget," Loersch \& Payne, 2011). In empirical evidence, conscious processes, such as need for achievement, mediated the relation between achievement goal priming and performance at a task (see review in Chen et al., 2020).

In summary, implicit goals align with explicit ones in guiding action in distinguishable ways from the direct cuing of habits. Implicit goals activate a flexible range of possible behaviors, establish a strategic orientation to achieve current desires, proceed in part through 
conscious inference, and have effects similar to explicit goals. Furthermore, direct empirical tests demonstrated that implicit goals do not account for habit persistence. These tests compellingly demonstrated that habits do not depend on goals, given that the studies assessed goals (a) broadly (e.g., liking for behaviors and outcomes), (b) personally nominated by participants, and (c) described in participants' own words. Given that participants with weak habits were guided by the relevant goals in these studies, the goal-independence of strong habits is not merely an artifact of measuring the wrong goals.

The direct context cuing of habits described in the preceding section reflects the procedural memory system underlying habitual responding. Two features of this memory system are especially relevant to distinguishing habits from goal-dependent responding. First, habits connect responses to context cues (places, people, preceding actions) associated with past performance, such that those responses are brought to mind automatically in those contexts. Second, the procedural memory system learns slowly and incrementally, making habit memory relatively resistant to short-term shifts in goals, preferences, and beliefs. We review evidence for each of these features below.

\section{Feature 1: Habitual Responses are Directly Activated by Past Performance Contexts}

The direct activation of habit responses enables speedy activation of the response from memory (Keramati et al., 2011). In evidence, participants extensively trained to respond with specific key presses to each of a series of pictures of Phoenician letters were then informed that the correct responses had changed and alternative keys were now correct (Hardwick et al., 2019). Although participants easily learned the new responses, when put under time pressure, they reverted back to responding out of habit (and against current goals). Nonetheless, given more time, participants could override their habits to act consistently with the new instructions 
(Hardwick et al., 2018). Thus, at short latencies, action was guided by habit memory and not current goals. At longer latencies, habits were masked by goal-dependent processes.

In an experiment that directly assessed the activation of habit responses and their effects on overt behavior, Labrecque et al. (2020) had participants practice a sequential task extensively or only twice. Showing habit activation, participants with extensive practice responded faster to an association test, which involved selecting the correct response after priming with a prior one in the sequence. A second part of the experiment then demonstrated the effects of response activation on overt behavior: Participants with shorter reaction times on the association test were able to more accurately repeat the full sequential task when under cognitive load. This study also demonstrated that effective performance did not depend on goals: The effects of direct associations between context cues and habitual responses emerged after statistically controlling for a number of goals that might vary with practice, such as intentions to accurately perform the task, fluency and ease of performance, and liking for the task.

Evidence of direct cuing in habit memory is not limited to novel lab tasks. Everyday habits are similarly activated quickly in mind (e.g., Danner et al., 2008). For example, frequent runners were faster to detect the words, "running" and "jogging," after being subliminally primed with the places where they typically ran (i.e., location-running associations, Neal et al., 2012). Providing additional evidence that habits are cued without goals, priming participants with their self-nominated goals for running did not activate thoughts of running for frequent runners. Thus, running habits were not linked to implicitly activated goals. Instead, goal priming was associated with running only for occasional runners, presumably because they still had to actively motivate themselves to run.

Research has thus demonstrated that greater practice, whether at experimental tasks or everyday behaviors, increases the speed with which people access responses when perceiving 
relevant context cues. Quick access to the response in mind gives habits precedence in action control (see Verbruggen and Logan's, 2008, classic horserace model of inhibition).

The activation of a particular response further advantages habits in action selection. People experience uncertainty and can fail to act when considering a large number of available options (Chernev et al., 2015). From a goal systems perspective, as the number of possible means increases, the available activation for each specific means decreases, forcing people to select among potential courses of action (Kruglanski et al., 2002). In fact, the narrowing of response options may be an important function of habits, as they constrain the broad set of responses that people could give at any moment (Morris et al., 2019).

In summary, habits often assume precedence in action control due to the speed of activation and the specificity of the response in mind. Furthermore, these appear to be a causal factor in the persistence of habitual responding (Labrecque et al., 2020). In the next section we consider a second principled implication of the definition of habit in current research.

\section{Feature 2: Habit Memory Is Insensitive to Newly Changed Goals and Beliefs}

Habit memory is a conservative action system that insulates repeated experience from short-term whims and transient shifts in people's goals and plans. Thus, unlike goals that energize and direct action flexibly in response to current desired ends (Kruglanski \& Szumowska, 2020), strong habits are recalcitrant and persist in memory regardless of whether they are consistent with current goals. Like procedural learning, or learning how to perform an action, habits develop gradually with practice and reflect the slow, incremental tuning of processing units in memory (Gupta \& Cohen, 2002). The saying that you never forget how to ride a bike illustrates this aspect of procedural/habit memories.

Illustrating this resilience, habit automaticity remained relatively stable even when people failed to act habitually in a given circumstance (Lally et al., 2010). Habit automaticity also 
persists despite people's intentions to act in other ways. For example, in the time-pressure research discussed earlier, habits continued to be activated despite participants' goal to override their habits (Hardwick et al., 2018). This effect has also been demonstrated in animal learning, in that habit memory decays only slowly and seems to maintain through reward extinction after animals have stopped responding (Adams, 1980; Bouton, 2019). Finally, computational models have successfully replicated the slow-learning feature of habit memory using simple Hebbian mechanisms that gradually connect contexts and responses in memory in ways that bypass goalmediated control of behavior (e.g., K. J. Miller et al., 2019).

In summary, two basic features of habit memory provide the underlying mechanism by which habits are cued directly by contexts without mediation by goals: Specific responses are quickly brought to mind by relevant contexts (Feature 1), and such habit memories endure despite changes in goals and failures to act (Feature 2). In the next section, we build on these features to identify the conditions in which people are likely to act on habit and ones in which they are likely to pursue goals. As we explain below, our predictions are supported by substantial empirical evidence.

\section{Moderators of Habit Performance}

If different psychological mechanisms guide habits as opposed to goal pursuit, then different conditions are likely to influence each type of action control. First, moderators that remove context cues should disrupt the process of habit performance while leaving the process of goal pursuit relatively intact. That is, for participants with strong habits, changes in cues should impede response activation so that the practiced response is not brought quickly to mind (see Feature 1). With a change in context, people with weaker habits might be spurred to pursue different goals, but these goals should continue to guide behavior through goal-based processes. Second, moderators that influence the process of goal pursuit should not disrupt the slow, 
incrementally changing habit memory trace (see Feature 2). Thus, a second test of our account is whether stressors, fatigue, and time pressures that impede goal processes leave habit processes relatively intact. Together these two moderator tests provide evidence of a kind of double dissociation, suggesting an underlying mental process that is required by habit but not by goals (and vice versa). As we noted above, this double dissociation logic has already been used to isolate the different neural systems associated with habit and declarative memory (Knowlton \& Diedrichsen, 2018).

Even clearer evidence of the separate processes of goal pursuit and habit performance comes from research using the same behavioral measure to demonstrate a double dissociation between habits and goals. Comparisons within a given behavioral domain are possible because habit refers to how a behavior is performed and not a type of response. Specifically, these studies identify one moderator of a given behavior that impedes performance for participants with strong habits but does not influence goal pursuit among those with weaker habits, along with a second moderator of that same behavior that impedes the influence of goals on participants with weak habits without affecting the performance of those with strong habits.

\section{Habit Is Altered By Context Change}

Studies of habit discontinuity reveal that habit performance is disrupted when people move to a new residence or start a new job (Verplanken, 2018; Verplanken et al, 2008). With the change in context, old cues no longer quickly activate the habitual response in mind (Feature 1), and behavior is more amenable to be guided by other action control processes, including goals. Thus, without cues to their everyday habits, new residents to an area were influenced more by a pro-environmental intervention than long-time residents whose living context had remained stable (Verplanken \& Roy, 2016). Suggesting habit's causal role, the same study found that habit 
strength uniquely predicted subsequent behavior over and above the contributions of goal-related constructs such as pro-environmental values and behavioral intentions.

A study of transfer students to a new university isolated the role of context cues in habit discontinuity (Wood et al., 2005). Replicating the classic discontinuity effect, when students' exercise contexts differed from the old to the new university, strong exercise habits were disrupted; students persisted in exercising only if they held strong exercise intentions. In addition, however, when contexts were stable across the move (e.g., indoor track at both schools), students maintained their exercise habit regardless of intentions. Given that exercise is beneficial in the long term but sometimes aversive in the short term, it might seem especially prone to the disruptive effects of context changes. However, the same pattern emerged with other, more immediately gratifying behaviors, such as TV watching. Just as with exercise, students' TV watching habits were disrupted when the transfer produced a change in the cues supporting this activity.

Controlled experiments have similarly demonstrated the moderating role of context cues on habit performance. For example, hungry participants were trained in an online task to choose carrots in response to an abstract image cue (Lin et al., 2016). Some were trained extensively so as to form a strong habit of choosing carrots when the specific cue was present. Others, with limited training, formed a weaker habit. All were subsequently given choices between carrots and M\&Ms. Strong habit participants were more likely to stick to their habitual (and healthy) choice on trials with the specific cue displayed on the screen, compared with trials without it. This was especially true for participants who reported high automaticity in their choice. Participants with weaker habits, in contrast, were more likely to choose M\&Ms regardless of the cue. This research challenges the folk wisdom that eating habitually is universally detrimental, and shows instead that healthy habits can protect people against choosing unhealthy foods. More 
relevant to the present point, the study varied an arbitrary cue (a fractal image) that is not plausibly associated with any change in eating goals. Thus, the change in participants' choices when habit cues changed cannot plausibly be traced to altered motives.

In summary, habit performance is disrupted when changes in contexts alter the cues that bring habits to mind. Regardless of whether the shift in cues was due to an altered living environment or to an experimental manipulation, the absence of habit cues leaves people acting in goal-dependent ways. When cues remained stable, however, people responded out of habit regardless of their personal intentions or the apparent desirability of the action. Furthermore, the parallel findings across everyday performance contexts and controlled experiments with artificial cues challenges goal-driven explanations in which context cues influenced the performance of strong habits by altering participants' goals.

\section{Time Pressure, Stress, and Fatigue Impede Goal Pursuit but not Habit}

Time limits, stress, and fatigue reduce people's capacity and motivation for deliberate goal pursuit while leaving habit memories intact. Each of these moderators impedes decisions through slightly different mechanisms, with time pressure limiting decision capacity, stress focusing processing on coping with the stressor, and fatigue reducing motivation more broadly.

We already discussed research in which time limits impeded pursuit of new, habitinconsistent goals (see section entitled, "Feature 1"). For example, under time pressure, participants expressed habitual responses regardless of whether they were correct, whereas with more time, they were able to prepare and express the correct response to meet current task goals (Hardwick et al., 2019). Also relevant, extensively learning an instrumental task under time pressure created response conflict, as participants' habitual responses continued to be activated even when the correct outcomes changed (Luque et al., 2020). The conflict was evident in 
reaction times to respond, and suggested that time pressure could encourage not only habit expression at the response stage but also habit formation at the learning stage.

\section{Stress}

In a review of stress effects on neural activation during performance of a variety of learning and memory tasks, Wirz et al. (2018) identified shifts from flexible, cognitively driven action control to more rigid habitual control. With navigation tasks, for example, stress induced a shift from the hippocampal system, which is associated with flexible and integrative learning, to the putamen and the sensorimotor cortico-basal ganglia network, which are involved in stimulus-response learning. Neurotransmitters activated by stressors (catecholamines, nongenomic glucocorticoids) help marshal cognitive resources to process the threat and to orient memory toward relying on habitual control (Quaedflieg \& Schwabe, 2018).

Illustrating the behavioral effects of stress, Schwabe and Wolf (2011) had previouslystressed and unstressed participants perform an instrumental task in which they repeatedly chose between two responses in order to get a reward. The task was easily learned regardless of stress. Rewards then were withheld on some blocks of trials (i.e., extinction). Stressed participants persisted in making the habitual choice even during these extinction trials. Those not stressed, in contrast, explored alternatives to get the reward. Ironically, by sticking with their habitual choice through the series of rewarded and unrewarded blocks of trials, stressed participants gained more rewards overall, while unstressed participants were exploring (unrewarded) alternatives even when the rewards started to be delivered again.

\section{Fatigue}

Research that experimentally manipulated fatigue also revealed that it impaired deliberate decisions despite leaving habit performance intact. In one study, for example, participants were mentally fatigued by using their nondominant hand for answering the phone and other activities 
(Neal et al., 2013). On days when they were fatigued in this way, participants continued to perform habitual, frequent behaviors but not more occasional actions, which presumably required effortful goal pursuit. The result of this differential moderation was that participants actually performed more habitual actions when fatigued than when rested. Importantly, habit performance increased across the board, including good habits that achieved participants' goals as well as bad ones that impeded goals. Thus, fatigue increased habit performance without dependence on goals.

Supporting this pattern of differential moderation, students fatigued by a difficult midterm exam were more likely to select snacks that were their usual, habitual choices than when they were not fatigued (Neal et al., 2013). Again, fatigue appeared to reduce deliberate control over behavior while leaving habit performance intact. The end result was that habit performance increased after the fatiguing exam. Importantly, fatigue did not affect motivational drivers of choice, including the perceived healthfulness of snacks or liking for them. Thus, fatigue increased habit performance without influencing motivations to act.

Additional evidence comes from a persuasion study in which participants did or did not complete a fatiguing task and then received a message on the health risks of sugar (Itzchakov et al., 2017). Fatigue increased persuasion so that all participants became less favorable to sugar consumption. However, when subsequently taste-testing beverages, fatigued participants with strong habits to drink sugared beverages continued to act habitually despite their new attitudes. In contrast, those not fatigued, much like all participants with weak beverage-drinking habits, acted on their new attitudes and avoided sampling sugared drinks. This study is especially informative because fatigue had reverse effects on habits and motivations--it decreased behavior change among strong habit participants while increasing their attitude change. 
In summary, stress and fatigue, much like extreme time pressure, reduce people's capacity to actively pursue current goals but have limited effect on habit memory. In fact, these moderators that impeded goal pursuit also increased the likelihood that people acted habitually. Furthermore, supporting the notion that habit automaticity does not depend on goals, people repeated both habits that yielded undesired outcomes as well as habits that generated desired ones.

\section{Doubly-Dissociated Moderators Within Behavioral Domain}

The strongest evidence that habitual control is distinct and independent from goaldependent control comes from demonstrations of multiple moderators of a given behavior-one moderator that influences habit performance but fails to impact goal pursuit, and another that influences goal pursuit but not habit performance. If independent moderators control habits and goals, then goals are not plausibly the underlying construct driving habit performance.

This double dissociation was evident in the strategies that people use in daily life to control unwanted responses (Quinn et al., 2010). In this experience sampling research, participants' control efforts included inhibiting strong habits, or actions performed repeatedly in stable contexts, as well as temptations, or behaviors motivated by immediate positive feelings despite longer-term regret. Participants successfully controlled habits by using a strategy of vigilant monitoring, or actively inhibiting acting on the response in mind. In contrast, participants effectively controlled temptations by distracting themselves. In a pattern of double dissociation, the habit-controlling strategy of vigilant monitoring had little impact on temptations, apparently because it increased focus on the tempting item, whereas the temptationcontrolling strategy of distraction had limited impact on habit performance, apparently because it allowed the habitual response to run off unattended. 
Posting on social media also revealed evidence of a double dissociation (Anderson \& Wood, 2020). Facebook users who posted only occasionally increased the frequency of their posts when they got more rewards from others in the form of likes and reactions. However, more frequent posters on the site, who respond automatically out of habit, did not increase posting when they got such rewards. In contrast, a change in the Facebook platform in 2008 changed context cues on the site, which decreased the rate at which frequent users posted messages, presumably because it disrupted habit automaticity. This same change, however, increased the frequency of posting for more occasional users.

Finally, eating research showed a double dissociation when experimentally manipulating liking for food as well as cues in the eating context (Neal et al., 2011). Specifically, patrons in a movie theater with strong habits to eat popcorn did so even when given stale popcorn that they reported disliking. In contrast, participants with weaker habits ate more fresh popcorn than stale. Thus, weak but not strong eating habits were responsive to motivation. A second study changed the context so that participants were given popcorn in a darkened meeting room on campus while watching movie videos. Participants with strong habits to eat popcorn at the cinema were no longer cued by the circumstances and responded much like weak habit participants, with everyone eating more fresh than stale popcorn. Thus, strong habits were most responsive to the change in context cues.

In evidence that eating habits were not goal-dependent, Neal et al. (2011) measured participants' hunger and their liking for the popcorn and demonstrated that strong habit participants were not guided by these motives any differently than those with weak habits. In this way, habits persisted without input from the most plausible goals that could drive behavior. Nonetheless, De Houwer (2019) speculated that strong habit participants persisted in eating stale popcorn because of yet another goal that had not been assessed in this study--that popcorn 
consumption was a valued part of movie-going for habitual but not nonhabitual participants. Of course, this value might be expected to influence reports of liking for the popcorn, but it did not. As noted earlier, however, this line of argument is unfalsifiable because an additional, as yet undiscovered, goal can always be proposed post hoc to explain habitual responding.

In summary, our definition of habit along with the features of habit memory isolate specific conditions under which habits and goals influence responding. When context cues shift, habits fail to be activated, and people are more likely to choose actions based on their current goals. Time pressure, fatigue, and stress disrupt slower, more effortful goal pursuit and leave people acting on habits, both goal-congruent and goal-incongruent. Finally, the clearest evidence for double dissociations occur within a single behavioral domain, as when factors that hinder goal pursuit leave habit performance relatively intact, whereas factors that disrupt habits leave people acting on goals. We reviewed these effects in three investigations involving the inhibition of everyday actions, posting on Facebook, and eating popcorn.

This evidence for the separate conditions that promote the processes of habit performance and goal pursuit challenges the claim that habits depend on goals. Yet separate moderators do not imply that habit performance is necessarily an alternative to goal pursuit. Although these two forms of action control implicate separate processes, they integrate in various ways to guide behavior (see Wood \& Neal, 2007; Wood \& Rünger, 2016). Especially performance of complex behaviors is likely to require coordination of habits and goals.

In the next section of the article, we provide a novel test of the characteristic features of habit memory. Specifically, we report a re-analysis of past research on action slips that had been interpreted as showing that habits are goal dependent (De Houwer et al., 2018). 
People experience action slips an average of six to seven times each week, according to experience sampling research (Jónsdóttir et al., 2007). Such slips often involve repeating unwanted habits when intending to do something else. This phenomenon is partly a reflection of Feature 2, in that habit memories are insensitive to short-term changes in goals. In a study of why consumers failed to follow through on their intentions to use newly-purchased products, 25\% said, "I fell back on my old habit and did what I used to do" (Labrecque et al., 2017).

Consistent with Feature 1, that habits are activated quickly, action slips are most likely when people are absentmindedly thinking about something unrelated to what they are doing (Reason, 2017). Thus, action slips are one consequence of the speed of habit activation and the persistence of habit memories despite new goals.

Our understanding of habit slips can potentially explain a paradox in recent research: If human habits do not depend on goals, then why has research failed to consistently demonstrate that they are insensitive to alterations in goals in outcome devaluation paradigms? As we noted earlier in discussing animal learning research, rodents extensively trained to perform a task for a reward continue to perform it even after the reward is devalued (animal is sated) or is no longer available (extinction, e.g., DeRusso et al., 2010; Dickinson 1985). This demonstration is often considered the gold standard indicator of habit formation (Wood \& Rünger, 2016).

Some, albeit limited, evidence that people respond habitually in outcome devaluation paradigms comes from neuroimaging research suggesting competing goal-dependent and habit systems (see review in de Wit et al., 2018). Furthermore, a broad body of findings from everyday life is consistent with the idea that strong habits persist despite people's ostensible goals (see reviews in Gardner, 2015; Mazar \& Wood, 2018; Wood \& Rünger, 2016). Additionally, the predicted pattern of behavioral persistence has emerged in a few controlled experiments. For example, participants trained extensively in an online task continued responding for a food 
reward even after they had eaten that food to the point of discomfort (Tricomi et al., 2009). However, a recent set of studies failed to find evidence that overtraining led to especially persistent responding after an outcome was devalued (de Wit et al., 2018).

To explain these inconsistent findings, some researchers have argued that outcome devaluation effects depend on goals (e.g., De Houwer, 2019; Kruglanski \& Szumowska, 2020). Supposedly, participants fail to respond to changes in outcomes not because they have formed a habit but because researchers have altered the wrong outcome. Stronger habit participants are supposedly pursuing an alternative goal or outcome than the one that was changed. However, this explanation requires that different goals drive strong and weak habit participants, given that those with weak habits do alter their behavior when outcomes are devaluated. This possibility is challenged by the comparable goals of strong and weak habit participants in similar paradigms (i.e., Lin et al., 2016; Neal et al., 2011).

De Houwer et al. (2018) nonetheless claimed to have tested the goal account by substituting a novel description of goals into an existing action-slip paradigm (de Wit et al., 2007; de Wit et al., 2012). In three studies, participants first repeatedly learned to associate cues (pictures of fruit) with responses (left or right key presses) that earned them points. After this training, participants were informed that responses that previously earned points would no longer do so, whereas alternative responses would now gain points. According to De Houwer et al. (2018), this instruction addressed participants' true goal in responding, which was to earn points, in contrast with the original studies, which framed the change in terms of correct and incorrect choices of fruits (de Wit et al., 2012). When informed about the point changes, De Houwer et al.'s (2018) extensively trained participants flexibly altered their responses, which supposedly indicated that they were driven by goals to acquire points and were not constrained by the 
practiced habitual choices. The continued sensitivity to point values held even for items designed to produce habitual responding, the incongruent items.

We reasoned, however, that overt behavior is not a sufficiently sensitive measure of habit effects. If habit memories are automatically activated by associated cues (Feature 1) and persist despite change in goals (Feature 2), then De Houwer et al.'s (2018) participants would have had to inhibit the now incorrect habitual response in order to pursue the goal of gaining points. Thus, on incongruent items, which were designed to form habits, they should demonstrate slower response times. Such a pattern would mirror findings from other paradigms in which inhibition manifests in slower reaction times even when overt responses are correct (e.g., Stroop task; MacLeod, 1992). In support, a recent set of studies measured both overt responses and reaction time in an outcome devaluation paradigm (Luque et al., 2020). As in De Houwer et al. and De Wit et al. (2018), participants were perfectly able to change their overt responses flexibly as outcome values shifted. However, in support of a habitual response component, response times on outcome-devalued trials were slower in conditions more likely to have created habits (greater training, greater time pressure, greater rewards during learning). Thus, it may be that previous studies did not observe habit effects because their outcome measure---overt responding---was not sensitive enough to capture habit.

To test this hypothesis, we obtained and analyzed the reaction time data from all three studies from De Houwer et al. (2018). Specifically, we evaluated whether, after the instructions that point values had changed, participants were slower to respond on the incongruent items, designed to promote habit formation, than on other types of items (congruent and biconditional), designed to reflect goal-dependent decisions. If habits were formed during the initial training on the habit items, then participants should respond more slowly due to inhibiting the incorrect response in mind and selecting the correct one. 
For the analysis, we constructed a multi-level linear model predicting reaction times from item type (effects coded, Habit $=1$, Non-habit $=-1)^{5}$, trial number following the instructions $(1$ 36), and interactions between trial and each item type (see multilevel model equations in Figure S1 in Supplemental Materials). Trials with response times more than three standard deviations slower than the mean were excluded from analyses (93 trials, or 3.2\% of trials). Nevertheless, the present findings persist when all observations are included. Raw data as well as code for these analyses can be found in the following link:

https://osf.io/zjw7t/?view_only=b69261b6394e499e83e4b99612fc0dfb.

\section{Figure 1}

Participants' Reaction Times Post Evaluation in De Houwer et al. (2018) as a Function of Trial Number and Item Type

${ }^{5}$ The present analysis collapsed across the two non-habit item types (congruent and biconditional). When analyzed separately, congruent items differed most from incongruent (habit) items, with biconditional items yielding results that fell between the other two types. Results were aggregated across all three studies to improve statistical power given the highly similar study designs. Power simulations for the single-study analyses using the simr package (Green \& MacLeod, 2016) suggested 68\% power for an interaction with a $t$ value of 4.1 (corresponding to a medium Cohen's $d$ effect size of 0.30), compared with $99 \%$ power for the analyses aggregating across studies. 


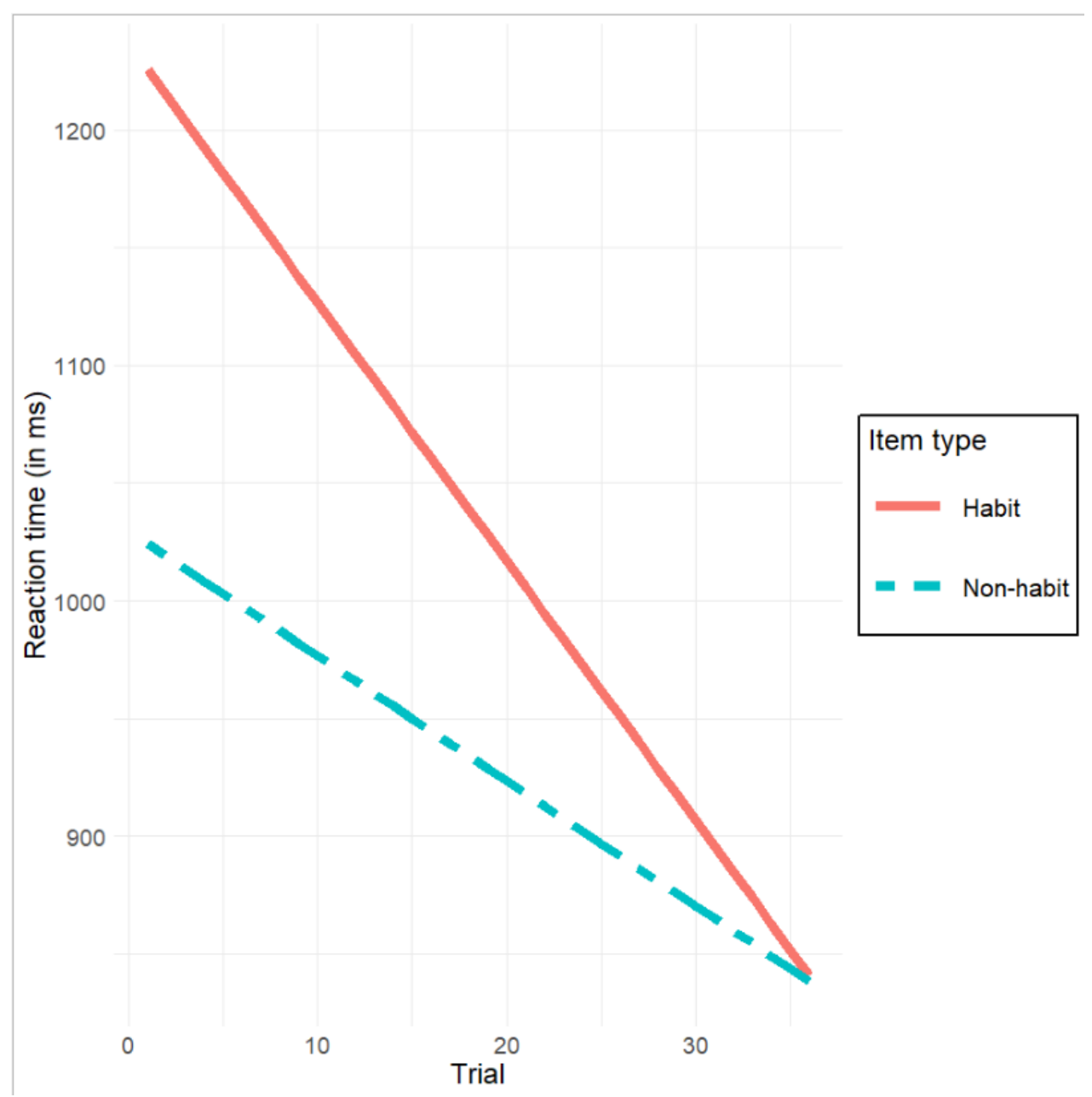

Note. The vertical axis reflects reaction times to respond during revaluation trials.

Regression results are illustrated in Figure 1. The analyses revealed a significant difference between habit and non-habit items, $b=103.54,95 \%$ CI $[64.40,142.68], t(2777)=$ $5.18, p<.001$, indicating that participants indeed gave slower responses on habit items than nonhabit items. A significant interaction between trial number and item type also emerged, $b=$ $2.84,95 \%$ CI $[-4.59,-1.10], t(2777)=-3.19, p<.001$, indicating that the difference between habit and non-habit items decreased with practice. A main effect of trial number, $b=-8.16,95 \%$ CI $[-9.90,-6.41], t(2777)=-9.15, p<.001$, demonstrated the expected decrease in reaction times on all items given practice. 
Thus, consistent with Feature 1, habits were quickly prepared on habit items even after the task rules were changed and participants were aware that acting on the habit would fail to gain points. Furthermore, consistent with Feature 2, the habitual memory trace persisted despite the change in participants' goals. However, with continued practice of the newly correct response across the 36 reevaluation trials, participants appear to have streamlined their responding to the task and equalized reaction times across incongruent items sensitive to habit formation and other items that were guided by goal pursuit.

Despite this evidence of the switch costs created by the automatic cuing of habit, De Houwer et al.'s (2018) study design may not be an optimal test. Detection of habits is challenged because all participants had extensive practice on all items. Even initially goal-directed actions can become habitual through sufficient repetition, and participants may have begun to develop habits for biconditional and congruent items. Further clouding interpretation, De Houwer et al.'s (2018) procedure deviated from the original outcome devaluation studies in more than just the changed goal description (e.g., reversal of all values, incorrect response losing points).

In sum, the paradox of outcome devaluation does not appear due to a failure for habits to persist when goals change so much as a failure for research assessments to be sensitive to habit activation. It seems that humans are almost as bedeviled by unwanted habits as are lab rats. However, with greater capacity to inhibit them, people are better able to control responses in action-slip or outcome-devaluation paradigms. As de Wit et al. (2018) speculated, failures to find evidence for action slips in experimental paradigms with humans could be due to participants' ability to deliberately override habits in settings that are not subject to stress, fatigue, or extreme time pressure. 
Despite the considerable evidence we reviewed showing that habits do not depend on goals, explaining behavior in terms of goals has broad appeal. In the next section, we show that goal accounts are so compelling, they can blind us to the workings of habit in everyday life.

\section{Inherent Plausibility of Goal Accounts}

Goal explanations have an inherent veneer of plausibility, both for people explaining everyday actions as well as for researchers. Such explanations are ubiquitous, with people readily able to infer goals to explain even the movement of dynamic geometric shapes, inferring that one shape was chasing another or trying to get away (Heider \& Simmel, 1944; Scholl \& Gao, 2013; Scholl \& Tremoulet, 2000). Accordingly, in controlled experiments, people explain their own and others' behavior primarily in terms of goals, whereas other potential causes, such as dispositions or situational constraints, are assumed to affect action indirectly via the goals that people are pursuing (Böhm \& Pfister, 2015). Such goal inferences are made spontaneously (Gilbert et al., 1988; Hassin et al., 2002) and are evident as early as 6.5 months old (Csibra, 2008). However, the prevalence and ease of goal-based attributions does not, of course, qualify as evidence that goals are casual drivers of habits.

We recently tested whether the plausibility of goal attributions might lead people to underestimate habit in their own behavior (Mazar \& Wood, 2020). In one study, coffee drinkers rated the extent to which their coffee drinking was driven by habit and by fatigue--the two most common explanations given in pilot testing. Then, over the course of one week, participants reported eight times per day on their current fatigue, context, and coffee drinking. Results showed that people drank coffee out of habit and fatigue, and these had comparably-sized effects. However, in participants' attributions, fatigue was a much stronger driver of their own coffee drinking than habit. This underestimation of habit persisted regardless of whether participants were making attributions (a) for themselves or others, (b) for their coffee drinking as 
a whole or for a single coffee drinking episode, and (c) when financially incentivized to provide accurate attributions for themselves.

In summary, people generally favor goal inferences, and these inferences feature prominently in lay explanations of behavior. Even when acting on habit, people overestimate the influence of goal pursuit and underestimate the influence of habit. The appeal of goal accounts may reflect the sense of agency and control that they establish for people in understanding of their own and others' actions. In our concluding section, we discuss the broader implications of continued research on habit formation and performance.

\section{Moving Forward}

In the present article, we showed that habits—-direct context-response associations learned through repeatedly rewarded responding — can account for important behavioral, cognitive, and neural phenomena in a principled way. Moreover, the findings we reviewed highlight the separate but interacting contributions of habit and goal processes in action control. Our integrative account offers predictive utility and avoids the non-falsifiability of post-hoc goal explanations.

Our analysis has implications for the research paradigms best suited to detect habits. Lab settings differ from everyday contexts in ways that often encourage goal-directed control over habit performance. For example, lab paradigms typically instruct participants to use one of a limited set of responses (e.g., left keypress or right keypress). Such designs contrast with the myriad actions possible at any given moment in daily life. Tellingly, in studies providing a free choice of food (rather than choice of one of two), participants' habits helped constrain the possible set to a small number of food choices (Morris et al, 2019). Additionally, experiments often provide incentives and sufficient time for thoughtful responding while limiting distraction and multitasking (e.g., phones, music). As we showed in our review of time pressures, stress, and 
fatigue, these features likely promote goal pursuit over habit responding. Furthermore, experiments may fail to create strongly habitual responses because of practical limitations on the number of repetitions.

Our point is not that habit researchers should abandon lab paradigms. Closely controlled settings can illuminate the mechanics of habit performance. Yet, experimental lab research provides a specific lens on human psychology that highlights certain processes over others (see Bless \& Burger, 2016; Yarkoni, 2019). In this vein, we encourage future researchers to be sensitive to the features that trigger and maintain habit performance that we identified in the present article. Thus, lab paradigms need to be structured with extensive repetition to build strong habits, recurring context cues to trigger them, along with time pressures and distractions to derail goal pursuit. Also useful are measures, like reaction time, that can track habit activation despite people's pursuit of goals (Luque et al., 2020).

Lab paradigms are best complemented by field and longitudinal methods to replicate realworld action control. To illustrate the payoff to a more naturalistic approach, we close with Rebar et al.'s (2014) elegant example of the interplay between exercise habits and goals in daily life: Over the course of two weeks, they measured the strength of participants' exercise habits, daily intentions to exercise, and actual physical exercise (via an accelerometer). On days when intentions to exercise were strong, habit strength did not predict physical activity, providing evidence for goal-directed control. However, on days when intentions to exercise were weak, stronger exercise habits led to greater physical activity. This longitudinal exploration highlights the functional utility of habits in maintaining behavior stability over time as goals inevitably fluctuate. We hope that the present article will encourage researchers to explore these separate but intersecting influences of habits and goals on human behavior. 


\section{References}

Adams, C. D. (1980). Post-conditioning devaluation of an instrumental reinforcer has no effect on extinction performance. Quarterly Journal of Experimental Psychology, 32(3), 447458. doi:10.1080/14640748008401838

Ainslie, G. (2020). Willpower with and without effort. Behavioral and Brain Sciences, 1-81. Advance online publication. doi:10.1017/S0140525X20000357

Amaya, K. A., \& Smith, K. S. (2018). Neurobiology of habit formation. Current Opinion in Behavioral Sciences, 20, 145-152. doi:10.1016/j.cobeha.2018.01.003

Anderson, I., \& Wood, W. (2020). Social media habits: When others' reactions don't matter. Manuscript in preparation.

Bargh, J. A. (in press). The cognitive unconscious in everyday life. To appear in A. Reber \& R. Allen (Eds.), The cognitive unconscious. Oxford University Press.

Bless, H., \& Burger, A. M. (2016). A closer look at social psychologists' silver bullet: Inevitable and evitable side effects of the experimental approach. Perspectives on Psychological Science, 11(2), 296-308. doi:10.1177/1745691615621278

Böhm, G., \& Pfister, H.-R. (2015). How people explain their own and others' behavior: A theory of lay causal explanations. Frontiers in Psychology, 6, Article 139. doi:10.3389/fpsyg.2015.00139

Botvinick, M. M., \& Plaut, D. C. (2004). Doing without schema hierarchies: A recurrent connectionist approach to normal and impaired routine sequential action. Psychological Review, 111(2), 395-429. doi:10.1037/0033-295X.111.2.395 
Bouton, M. E. (2019). Extinction of instrumental (operant) learning: Interference, varieties of context, and mechanisms of contextual control. Psychopharmacology, 236, 7-19. doi:10.1007/s00213-018-5076-4

Campbell, D. T. (1963). Social attitudes and other acquired behavioral dispositions. In S. Koch, Psychology: A study of a science. Study II. Empirical substructure and relations with other sciences. Vol. 6. Investigations of man as socius: Their place in psychology and the social sciences (p. 94-172). McGraw-Hill. doi:10.1037/10590-003

Carver, C. S., \& Scheier, M. F. (1982). Control theory: A useful conceptual framework for personality-social, clinical, and health psychology. Psychological Bulletin, 92(1), 111135. doi:10.1037/0033-2909.92.1.111.

Chen, X., Latham, G. P., Piccolo, R. F., \& Itzchakov, G. (2020). An enumerative review and a meta-analysis of primed goal effects on organizational behavior. Applied Psychology. doi:10.1111/apps.12239

Chernev, A., Böckenholt, U., \& Goodman, J. (2015). Choice overload: A conceptual review and meta-analysis. Journal of Consumer Psychology, 25(2), 333-358. doi:10.1016/j.jpps.2014.08.002

Corbit, L. H. (2018). Understanding the balance between goal-directed and habitual behavioral control. Current Opinion in Behavioral Sciences, 20, 161-168. doi:10.1016/j.cobeha.2018.01.010

Csibra, G. (2008). Goal attribution to inanimate agents by 6.5-month-old infants. Cognition, 107(2), 705-717. doi:10.1016/j.cognition.2007.08.001

Danner, U. N., Aarts, H., \& de Vries, N. K. (2008). Habit vs. intention in the prediction of future behaviour: The role of frequency, context stability and mental accessibility of past 
behaviour. British Journal of Social Psychology, 47(2), 245-265.

doi:10.1348/014466607X230876

De Houwer, J. (2019). On how definitions of habits can complicate habit research. Frontiers in Psychology, 10, Article 2642. doi:10.3389/fpsyg.2019.02642

De Houwer, J., Tanaka, A., Moors, A., \& Tibboel, H. (2018). Kicking the habit: Why evidence for habits in humans might be overestimated. Motivation Science, 4(1), 50-59. doi:10.1037/mot0000065

DeRusso, A. L., Fan, D., Gupta, J., Shelest, O., Costa, R. M., \& Yin, H. H. (2010). Instrumental uncertainty as a determinant of behavior under interval schedules of reinforcement. Frontiers in Integrative Neuroscience, 4, Article 17. doi:10.3389/fnint.2010.00017 de Wit, S. (2017). Control of behaviour by competing learning systems. In T. Edgar (Ed.), The Wiley handbook of cognitive control (p. 190-206). Wiley Blackwell. doi:10.1002/9781118920497.ch11

de Wit, S., Niry, D., Wariyar, R., Aitken, M. R. F., \& Dickinson, A. (2007). Stimulus-outcome interactions during instrumental discrimination learning by rats and humans. Journal of Experimental Psychology: Animal Behavior Processes, 33(1), 1-11. doi:10.1037/00977403.33.1.1

de Wit, S., Watson, P., Harsay, H. A., Cohen, M. X., van de Vijver, I., \& Ridderinkhof, K. R. (2012). Corticostriatal connectivity underlies individual differences in the balance between habitual and goal-directed action control. Journal of Neuroscience, 32(35), 12066-12075. doi:10.1523/JNEUROSCI.1088-12.2012

de Wit, S., Kindt, M., Knot, S. L., Verhoeven, A. A., Robbins, T. W., Gasull-Camos, J., ... \& Gillan, C. M. (2018). Shifting the balance between goals and habits: Five failures in 
experimental habit induction. Journal of Experimental Psychology: General, 147(7), 1043-1065. doi:10.1037/xge0000402

Dezfouli, A., \& Balleine, B. W. (2013). Actions, action sequences and habits: Evidence that goal-directed and habitual action control are hierarchically organized. PLOS Computational Biology, 9(12), Article e1003364. doi:10.1371/journal.pcbi.1003364

Dickinson, A. (1985). Actions and habits: The development of behavioural autonomy. Philosophical Transactions of the Royal Society of London. B, Biological Sciences, 308(1135), 67-78. doi:10.1098/rstb.1985.0010

Dolan, R., \& Dayan, P. (2013). Goals and habits in the brain. Neuron, 80(2), 312-325. doi:10.1016/j.neuron.2013.09.007

Ersche, K. D., Lim, T. V., Murley, A. G., Rua, C., Vaghi, M. M., White, T. L., Williams, G. B., \& Robbins, T. W. (in press). Reduced glutamate turnover in the putamen is linked with automatic habits in human cocaine addiction. Biological Psychiatry.

Foerde, K. (2018). What are habits and do they depend on the striatum? A view from the study of neuropsychological populations. Current Opinion in Behavioral Sciences, 20, 17-24. doi:10.1016/j.cobeha.2017.08.011

Gardner, B. (2015). A review and analysis of the use of 'habit' in understanding, predicting and influencing health-related behaviour. Health Psychology Review, 9(3), 277-295. doi:10.1080/17437199.2013.876238

Giesen, C. G., Schmidt, J. R., \& Rothermund, K. (2019). The law of recency: An episodic stimulus-response retrieval account of habit acquisition. Frontiers in Psychology, 10, Article 2927. doi:10.3389/fpsyg.2019.02927 
Gilbert, D. T., Pelham, B. W., \& Krull, D. S. (1988). On cognitive busyness: When person perceivers meet persons perceived. Journal of Personality and Social Psychology, 54(5), 733-740. doi:10.1037/0022-3514.54.5.733

Green, P., \& MacLeod, C. J. (2016). “simr: an R package for power analysis of generalised linear mixed models by simulation." Methods in Ecology and Evolution, 7(4), 493-498. doi: 10.1111/2041-210X.12504, https://CRAN.R-project.org/package=simr.

Gupta, P., \& Cohen, N. J. (2002). Theoretical and computational analysis of skill learning, repetition priming, and procedural memory. Psychological Review, 109(2), 401-448. doi:10.1037//0033-295X.109.2.401

Haith, A. M., \& Krakauer, J. W. (2018). The multiple effects of practice: Skill, habit and reduced cognitive load. Current Opinion in Behavioral Sciences, 20, 196-201. doi:10.1016/j.cobeha.2018.01.015

Hassin, R. R., Bargh, J. A., \& Uleman, J. S. (2002). Spontaneous causal inferences. Journal of Experimental Social Psychology, 38(5), 515-522. doi:10.1016/S0022-1031(02)00016-1

Hassin, R. R., Bargh, J. A., \& Zimerman, S. (2009). Automatic and flexible: The case of nonconscious goal pursuit. Social Cognition, 27(1), 20-36. doi:10.1521/soco.2009.27.1.20

Hardwick, R. M., Forrence, A. D., Krakauer, J. W., \& Haith, A. M. (2019). Time-dependent competition between goal-directed and habitual response preparation. Nature Human Behaviour, 3, 1252-1262. doi:10.1038/s41562-019-0725-0

Heider, F., \& Simmel, M. (1944). An experimental study of apparent behavior. American Journal of Psychology, 57(2), 243-249. https://doi.org/10.2307/1416950 
Hull, C. L. (1943). Principles of behavior: An introduction to behavior theory. New York, NY: Appleton-Century-Crofts.

Hommel, B. (2019). Binary theorizing does not account for action control. Frontiers in Psychology, 10, Article 2542. doi:10.3389/fpsyg.2019.02542

Itzchakov, G., Uziel, L., \& Wood, W. (2018). When attitudes and habits don't correspond: Selfcontrol depletion increases persuasion but not behavior. Journal of Experimental Social Psychology, 75, 1-10. doi:10.1016/j.jesp.2017.10.011

James, W. (1898). The principles of psychology. New York, NY: Holt.

James, W. (1914). Habit. New York, NY: Holt.

Ji, M. F., \& Wood, W. (2007). Purchase and consumption habits: Not necessarily what you intend. Journal of Consumer Psychology, 17(4), 261-276. doi:10.1016/S10577408(07)70037-2

Jónsdóttir, M. K., Adólfsdóttir, S., Cortez, R. D., Gunnarsdóttir, M., \& Gústafsdóttir, Á. H. (2007). A diary study of action slips in healthy individuals. The Clinical Neuropsychologist, 21(6), 875-883. doi:10.1080/13854040701220044

Keramati, M., Dezfouli, A., \& Piray, P. (2011). Speed/accuracy trade-off between the habitual and the goal-directed processes. PLoS Computational Biology, 7(5), Article e1002055. doi:10.1371/journal.pcbi.1002055

Knowlton, B. J., \& Diedrichsen, J. (2018). Editorial overview: Habits and skills. Current Opinion in Behavioral Sciences, 20, iv-vi. doi:10.1016/j.cobeha.2018.02.009

Kruglanski, A. W., Shah, J. Y., Fishbach, A., Friedman, R., Chun, W. Y., \& Sleeth-Keppler, D. (2002). A theory of goal systems. In Advances in Experimental Social Psychology (Vol. 34, p. 331-378). Academic Press. 
Kruglanski, A. W., \& Szumowska, E. (2020). Habitual behavior is goal-driven. Perspectives on Psychological Science, 15(5), 1256-1271. doi:10.1177/1745691620917676

Labrecque, J. S., Lee, K., \& Wood, W. (2020). How habits stick. Under editorial review.

Labrecque, J. S., Wood, W., Neal, D. T., \& Harrington, N. (2017). Habit slips: When consumers unintentionally resist new products. Journal of the Academy of Marketing Science, 45, 119-133. doi:10.1007/s11747-016-0482-9

Lally, P., Van Jaarsveld, C. H., Potts, H. W., \& Wardle, J. (2010). How are habits formed: Modelling habit formation in the real world. European Journal of Social Psychology, 40(6), 998-1009. doi:10.1002/ejsp.674

Lin, P. Y., Wood, W., \& Monterosso, J. (2016). Healthy eating habits protect against temptations. Appetite, 103, 432-440. doi:10.1016/j.appet.2015.11.011

Loersch, C., \& Payne, B. K. (2011). The situated inference model: An integrative account of the effects of primes on perception, behavior, and motivation. Perspectives on Psychological Science, 6(3), 234-252. doi:10.1177/1745691611406921

Luque, D., Molinero, S., Watson, P., López, F. J., \& Le Pelley, M. E. (2020). Measuring habit formation through goal-directed response switching. Journal of Experimental Psychology: General, 149(8), 1449-1459. http://dx.doi.org.libproxy1.usc.edu/10.1037/xge0000722

Mazar, A., \& Wood, W. (2018). Defining habit in psychology. In B. Verplanken (Ed.), The psychology of habit: Theory, mechanisms, change, and context (p. 13-29). Cham, Switzerland: Springer. doi:10.1007/978-3-319-97529-0_2

Mazar, A., \& Wood, W. (2020). Illusory motives, elusive habits: Lay theories of everyday behavior. Manuscript in preparation. 
MacLeod, C. M. (1992). The Stroop task: The "gold standard" of attentional measures. Journal of Experimental Psychology: General, 121(1), 12-14. doi:10.1037/0096-3445.121.1.12

Miller, G. A., Galanter, E., \& Pribram, K. H. (1960). Plans and the structure of behavior. New York, NY: Henry Holt. doi:10.1037/10039-000

Miller, K. J., Shenhav, A., \& Ludvig, E. A. (2019). Habits without values. Psychological Review, 126(2), 292-311. doi:10.1037/rev0000120

Moors, A., \& De Houwer, J. (2006). Automaticity: A theoretical and conceptual analysis. Psychological Bulletin, 132(2), 297-326. doi:10.1037/0033-2909.132.2.297

Morris, A., Phillips, J. S., Huang, K., \& Cushman, F. (2019). Habits of thought generate candidate actions for choice. PsyArXiv. doi:10.31234/osf.io/2ayw3

Neal, D. T., Wood, W., \& Drolet, A. (2013). How do people adhere to goals when willpower is low? The profits (and pitfalls) of strong habits. Journal of Personality and Social Psychology, 104(6), 959-975. doi:10.1037/a0032626

Neal, D. T., Wood, W., Labrecque, J. S., \& Lally, P. (2012). How do habits guide behavior? Perceived and actual triggers of habits in daily life. Journal of Experimental Social Psychology, 48(2), 492-498. doi:10.1016/j.jesp.2011.10.011

Neal, D. T., Wood, W., Wu, M., \& Kurlander, D. (2011). The pull of the past: When do habits persist despite conflict with motives?. Personality and Social Psychology Bulletin, 37(11), 1428-1437. doi:10.1177/0146167211419863

O’Hare, J., Calakos, N., \& Yin, H. H. (2018). Recent insights into corticostriatal circuit mechanisms underlying habits. Current Opinion in Behavioral Sciences, 20, 40-46. doi:10.1016/j.cobeha.2017.10.001 
O'Reilly, R. C., Nair, A., Russin, J. L., \& Herd, S. A. (2020). How sequential interactive processing within frontostriatal loops supports a continuum of habitual to controlled processing. Frontiers in Psychology, 11, Article 380. doi:10.3389/fpsyg.2020.00380

Ouellette, J. A., \& Wood, W. (1998). Habit and intention in everyday life: The multiple processes by which past behavior predicts future behavior. Psychological Bulletin, 124(1), 54-74. doi:10.1037/0033-2909.124.1.54

Patterson, T. K., \& Knowlton, B. J. (2018). Subregional specificity in human striatal habit learning: A meta-analytic review of the fMRI literature. Current Opinion in Behavioral Sciences, 20, 75-82. doi:10.1016/j.cobeha.2017.10.005

Quaedflieg, C. W., \& Schwabe, L. (2018). Memory dynamics under stress. Memory, 26(3), 364376. doi:10.1080/09658211.2017.1338299

Quinn, J. M., Pascoe, A., Wood, W., \& Neal, D. T. (2010). Can’t control yourself? Monitor those bad habits. Personality and Social Psychology Bulletin, 36(4), 499-511. doi:10.1177/0146167209360665

Reason, J. (2017). A life in error: From little slips to big disasters. New York, NY: CRC Press.

Rebar, A. L., Elavsky, S., Maher, J. P., Doerksen, S. E., \& Conroy, D. E. (2014). Habits predict physical activity on days when intentions are weak. Journal of Sport and Exercise Psychology, 36(2), 157-165. doi:10.1123/jsep.2013-0173

Scholl, B. J., \& Gao, T. (2013). Perceiving animacy and intentionality: Visual processing or higher-level judgment? In M. D. Rutherford \& V. A. Kuhlmeier (Eds.), Social perception: Detection and interpretation of animacy, agency, and intention (pp. 197230). MIT Press. doi:10.7551/mitpress/9780262019279.003.0009 
Scholl, B. J., \& Tremoulet, P. D. (2000). Perceptual causality and animacy. Trends in Cognitive Sciences, 4(8), 299-309. doi:10.1016/S1364-6613(00)01506-0

Schwabe, L., \& Wolf, O. T. (2011). Stress increases behavioral resistance to extinction. Psychoneuroendocrinology 36(9), 1287-1293. doi:10.1016/j.psyneuen.2011.02.002

Skinner, B. F. (1938). The behavior of organisms: An experimental analysis. New York, NY: Appleton-Century.

Sun, R., Slusarz, P., \& Terry, C. (2005). The interaction of the explicit and the implicit in skill learning: A dual-process approach. Psychological Review, 112(1), 159-192. doi:10.1037/0033-295X.112.1.159

Takarada, Y., \& Nozaki, D. (2018). Motivational goal-priming with or without awareness produces faster and stronger force exertion. Scientific Reports, 8, Article 10135. doi:10.1038/s41598-018-28410-0

Tolman, E. C. (1948). Cognitive maps in rats and men. Psychological Review, 55(4), 189-208. doi:10.1037/h0061626

Triandis, H. C. (1977). Interpersonal behavior. Monterey, CA: Brooks/Cole.

Tricomi, E., Balleine, B. W., \& O’Doherty, J. P. (2009). A specific role for posterior dorsolateral striatum in human habit learning. European Journal of Neuroscience, 29(11), 2225-2232. doi:10.1111/j.1460-9568.2009.06796.x

Verbruggen, F., \& Logan, G. D. (2008). Response inhibition in the stop-signal paradigm. Trends in Cognitive Sciences, 12(11), 418-424. doi:10.1016/j.tics.2008.07.005

Verplanken, B. (2018). The psychology of habit: Theory, mechanisms, change, and contexts. Switzerland: Springer Nature. 
Verplanken, B., Walker, I., Davis, A., \& Jurasek, M. (2008). Context change and travel mode choice: Combining the habit discontinuity and self-activation hypotheses. Journal of Environmental Psychology, 28(2), 121-127. doi:10.1016/j.jenvp.2007.10.005

Verplanken, B., \& Roy, D. (2016). Empowering interventions to promote sustainable lifestyles: Testing the habit discontinuity hypothesis in a field experiment. Journal of Environmental Psychology, 45, 127-134. doi:10.1016/j.jenvp.2015.11.008

Wiltgen, B. J., Sinclair, C., Lane, C., Barrows, F., Molina, M., \& Chabanon-Hicks, C. (2012). The effect of ratio and interval training on Pavlovian-instrumental transfer in mice. PloS one, 7(10), Article e48227. doi:10.1371/journal.pone.0048227

Wirz, L., Bogdanov, M., \& Schwabe, L. (2018). Habits under stress: Mechanistic insights across different types of learning. Current Opinion in Behavioral Sciences, 20, 9-16. doi:10.1016/j.cobeha.2017.08.009

Wood, W., \& Neal, D. T. (2007). A new look at habits and the habit-goal interface. Psychological Review, 114(4), 843-863. doi:10.1037/0033-295X.114.4.843

Wood, W., \& Rünger, D. (2016). Psychology of habit. Annual Review of Psychology, 67, 289314. doi:10.1146/annurev-psych-122414-033417

Wood, W., Tam, L., \& Witt, M. G. (2005). Changing circumstances, disrupting habits. Journal of Personality and Social Psychology, 88(6), 918-933. doi:10.1037/0022-3514.88.6.918

Yarkoni, T. (2019). The generalizability crisis. PsyArXiv. doi:10.31234/osf.io/jqw35

Yin, H. H., \& Knowlton, B. J. (2006). The role of the basal ganglia in habit formation. Nature Reviews Neuroscience, 7, 464-476. doi:10.1038/nrn1919 